\title{
Beyond Ricardian Model: An Optimal Commodity Distribution Based on Absolute Advantage for Multi-Country Multi-Commodity
}

\author{
Eun Ju Lee ${ }^{1}$, Yeongseop Rhee ${ }^{1} \&$ Sang-Un Lee ${ }^{2}$ \\ ${ }^{1}$ Graduate School of International Studies, Seoul National University, Seoul, South Korea \\ ${ }^{2}$ Department of Multimedia Engineering, Gangneung-Wonju National University, Wonju, South Korea \\ Correspondence: Eun Ju Lee, Graduate School of International Studies, Seoul National University, Seoul, \\ 151-742, South Korea. E-mail: leighannechoo@snu.ac.kr
}

Received: May 12, 2013 Accepted: June 8, 2013 Online Published: June 18, 2013

doi:10.5539/ijbm.v8n14p110 URL: http://dx.doi.org/10.5539/ijbm.v8n14p110

\begin{abstract}
Ricardo's comparative advantage model asserts that international trade will make every single participant country better off if they traded goods in which they have comparative advantage. Tian (2008) has generalized and expanded this Ricardo's 2-country, 2-commodity comparative advantage into a multi-country, multi-commodity model. His methodology, however, occasionally fails to achieve optimal commodity distribution or to facilitate international trade even if it succeeds in optimal distribution, for it results in a high degree of difference in countries' commodity prices. This paper proposes an algorithm that selects countries' comparatively advantageous goods for multi-country, multi-commodity model based on their absolute advantage. The proposed algorithm simply selects a commodity with the maximum absolute advantage - the minimum price - from each country and reassigns commodities of over-assigned countries to under-assigned countries. This absolute advantage model (AAA) is found to be much simpler than Tian's comparative advantage method and to yield superior results.
\end{abstract}

Keywords: comparative advantage, absolute advantage, sum price rate, average price rate, multi-country multi-commodity

\section{Introduction}

In international trade involving two nations, although one country possesses absolute advantage in all goods (i.e., has higher efficiency in the production of all goods), both participant countries could still gain by trading with each other, so long as they have different relative efficiencies (O'Sullivan and Sheffrin, 2003; Baumol, 2009). This concept is called the law of comparative advantage, and it refers to the ability of a party to produce a particular good or service at a lower opportunity cost than another.

Comparative advantage was first developed by David Ricardo (1817) who explained it in his book On the Principles of Political Economy and Taxation to explain a possible trade pattern between two countries (England and Portugal) involving two commodities (cloth and wine). This optimal commodity distribution model, dating back almost 200 years, has been bound to a 2-country, 2-commodity matrix. This triggered several researches that have attempted to expand Ricardo's 2-country, 2-commodity comparative advantage model into a multi-country, multi-commodity model: namely Tian's model (Tian, 2008) and Graham's theory (Parchure, 2011). Tian's model (Tian, 2008) is based on the commodity price, and Graham's theory (Parchure, 2011) on a number of variables such as labor cost, wage rate, and exchange rate. This paper only considers the commodity price as Tian's model does. Tian's model repeatedly computes average price rates (apr) and selects the maximum comparative advantage - the smallest average price rate. This model, however, occasionally fails to obtain the optimal value or to facilitate international trade even if it succeeds in optimal distribution, for the difference in countries' commodity prices is large. Additionally, in the existence of multiple commodities with equal maximum comparative advantage, it yields different outcomes depending on which commodity it selects.

To remedy the shortcomings of Tian's apr-CA model, this paper proposes an algorithm that selects a commodity with the maximum absolute advantage - the lowest price - for each country and reassigns commodities of over-assigned countries to under-assigned countries. In chapter 2, we review Tian's apr-CA model for optimal commodity distribution in a multi-country, multi-commodity setting and discuss its limitations. In chapter 3 , we 
propose an optimal commodity distribution algorithm based on absolute advantage (AA), which we name the Absolute Advantage Algorithm (AAA). In chapter 4, we compare the performances of Tian's apr-CA model with those of AAA on various commodity distribution cases.

\section{Related Studies and Problems of an M-Country and N-Commodity Model}

Although the Ricardian model's comparative advantage concept has been formulated for and confined to a 2-country, 2-commodity case, it could easily be extended to a 2-country, multi-commodity case or a multi-country, 2-commodity case (Deardorff, 2005). However, it is not as easy to generalize and expand the model to a pure multi-country, multi-commodity case. Tian's model has nevertheless attempted to generalize this 2-country, 2-commodity comparative advantage model into a multi-country, multi-commodity model. (Tian, 2008) For a better understanding of this model, let's examine how Tian has generalized Ricardo's comparative advantage and expanded it into the multi-country multi-commodity model.

Let $m$-Country, $i=1,2, \cdots, m$ and $n$-Commodity, $j=1,2, \cdots, n$.

In generalizing Ricardo's comparative advantage, one may derive the equation for sum price rate $r_{s(i, j)}$ for $i^{\text {th }}$-country, $j^{\text {th }}$-commodity as shown in equation (1).

$$
\begin{gathered}
\text { Sum price rate: } r_{s(i, j)}=\frac{P_{(i, j)}}{\left(\sum_{i=1}^{m} P_{(i, j)}-P_{(i, j)}\right)} \\
\text { where } \left.\sum_{i=1}^{m} P_{(i, j)}-P_{(i, j)}\right) \text { for }|i| \geq 2 \\
P_{(i, j)} \text { for }|i|=1
\end{gathered}
$$

Tian (2008) on the other hand proposed an average price rate model for a multi-country and multi-commodity case based on Ricardo's comparative advantage model as shown in equation (2).

$$
\text { Average price rate: } r_{a(i, j)}=\frac{P_{(i, j)}}{\left(\sum_{i=1}^{m} P_{(i, j)}-P_{(i, j)}\right) /(n-1)}
$$

Tian (2008) computes and takes into the consideration the average price of identical commodities of all other countries instead of their sum price in calculating the price rate of a commodity produced by a country, and selects the optimal commodity as Ricardian model does in the comparative advantage model. Tian thus transforms an $n \times n$ matrix of nominal prices into an $n \times n$ matrix of average price rates.

Subsequently, Tian selects the minimum average price rate from the $n \times n$ matrix of average price rates and obtains a new $(n-1) \times(n-1)$ matrix of prices, derived from deleting the column and the row of the selected commodity possessing the minimum average price rate. This is then converted into an $(n-1) \times(n-1)$ matrix of average price rates and the minimum average price rate is selected once again. This process is repeated until it transforms the original matrix into a $1 \times 1$ price set, leaving all $n$ countries allocated with a single commodity to specialize in.

Tian's apr-CA model of commodity distribution, however, has several drawbacks as follows:

(1) apr-CA model executes $n^{3}+(n-1)^{3}+(n-2)^{3}+\cdots+2^{3}=\left(\frac{n(n+1)}{2}\right)^{2}-1$ times the computation of the average price rates; $n^{2}+(n-1)^{2}+(n-2)^{2}+\cdots+2^{2}+1=\frac{n(n+1)(2 n+1)}{6}$ times the selection of the minimum average price rates; and $2(n+(n-1)+\cdots+2+1)=n(n+1)$ times the deletion of each row and column, making it a highly complicated process. As the values of $m$ and $n$ increase, so would the complexity by multifold.

(2) apr-CA model is a 'Greedy Algorithm', i.e., it selects a good with the maximum comparative advantage at that specific point in time with no regard whatsoever to the price rates of the rest of the commodities. This type of method could yield one of the following three results:

- A case where countries are left with extreme values although the sum price of the selected commodities turns out optimal provided that the difference between the minimum cost and the maximum cost is large. Put another way, some countries will be left specializing in commodities in which they have the best price competitiveness and others in commodities in which they have the worst price competitiveness, driving the latter countries out of the competition in the arena of international trade. Let's term this an 'unsatisfyingly accepted case' (or interchangeably a 'nominal case'); 
- A case where the sum price of the selected commodities is optimal and the difference between the minimum cost and the maximum cost is minimal such that international trade could be proliferated. Let's term this a 'satisfyingly accepted case' (or interchangeably the 'best case'); or

- A case where the sum price of the selected commodities fails to be optimal. Let's call this a 'rejected case' (or interchangeably the 'worst case'). In the presence of multiple commodities with equal maximum comparative advantage, Tian's model could yield highly different results depending on a commodity it selects

(3) apr-CA model could only be applied when $m=n$, not when $m \neq n(m<n)$. Let's term $m=n$ as a 'balanced distribution' and $m \neq n(m<n)$ an 'unbalanced distribution'.

In the following chapter, we propose an alternate algorithm based on absolute advantage for $m$-country, $n$-commodity where $m=n$ and alsowhere $m \neq n(m<n)$.

\section{Absolute Advantage Model for Multi-Country and Multi-Commodity}

In this chapter, we propose a simple algorithm that could be universally applied to any given multi-country, multi-commodity model, be it a balanced distribution, where $m=n$ or an unbalanced distribution, where $m \neq n(m<n)$. Whereas Tian'sapr-CA model is based on comparative advantage (average price rates), the proposed algorithm is solely based on absolute advantage - relying on nominal prices. We name this algorithm an absolute advantage algorithm (AAA).

Amongst rows in this algorithm, we make set $A_{o}$ stand for an over-assigned set, set $A_{u}$ for an under-assigned set, and set $A_{n}$ for a normally-assigned set.

Step 1. Select the minimum price, $\min P_{(i, j)}$ from each column $(j=1,2, \cdots, n)$.

Step 2. Identify $A_{o}, A_{u}$, and $A_{n}$ for each row $(i=1,2, \cdots, m)$

Step 3. If $\left|A_{n}\right|=m$, where $\left|A_{n}\right|$ is the cardinality of $A_{n}$, the optimal value is obtained and thus the algorithm comes to an end.

If, however, $\left|A_{u}\right|>0$ and $\left|A_{o}\right|>0$, an excess commodity must be shifted from $A_{o}$ to $A_{u}$. In this case, there are two options. One is a direct moving path where a commodity is moved directly from $A_{o}$ to $A_{u}$. The other is an indirect moving path where an excess commodity of $A_{o}$ is shifted to $A_{n}$, and again a commodity belonging to $A_{n}$, which is pricier than that of $A_{o}$ by the smallest margin, is moved from $A_{n}$ to $\mathrm{A}_{u}$. Here, we employ whichever method that entails the minimum price increase. If both methods result in an equal price increase, we move first the commodity with the minimum price from $\mathrm{A}_{o}$ and leave the commodity with the maximum price fixed. This process is repeated until $\left|A_{u}\right|=0$. In cases where $m \leq k n$, we leave as many commodities as $k$ for each country and reassign excessively assigned commodities.

This would allow this algorithm to be applied in all cases whether $m=n$ or $m \neq n(m<n)$.

The proposed AAA executes $n \times n=n^{2}$ times the selection of the minimum prices and at most $n \times n=n^{2}$ times the rearrangement of commodities from over-assigned countries to under-assigned countries, hence $2 n^{2}$ times for the total process. This algorithm could therefore simplify the optimal commodity distribution process of Tian's $\left(\frac{n(n+1)}{2}\right)^{2}+\frac{n(n+1)(2 n+1)}{6}+n(n+1)-1$ times into $2 n^{2}$ times. Additionally, despite its relative simplicity, it yields superior results in optimal commodity distribution.

Numerous past researches on optimal commodity distribution have only adopted the concept of comparative advantage. This paper proposes the first algorithm based on absolute advantage and simultaneously succeeds in bringing about better results than do existing algorithms, which have applied the concept of comparative advantage.

\section{Experimental Study and Result Analysis}

Table 1 demonstrates test results of apr-CA model and AAA on the case data among which $\mathrm{BD}_{1}$ and $\mathrm{UBD}_{1}$ have been directly quoted from Tian (Tian, 2008). As shown in the 'Case of Result' columns, whereas AAA has achieved 'Accepted' for all the case data, apr-CA model has obtained 'Rejected' for $\mathrm{BD}_{2}, \mathrm{BD}_{4}, \mathrm{BD}_{7}$ and $\mathrm{UBD}_{4}$, and 'Nominal' for $\mathrm{BD}_{5}, \mathrm{BD}_{6}$ and $\mathrm{UBD}_{2}$. Moreover, apr-CA model faced selection dilemma in $\mathrm{BD}_{2}$ and $\mathrm{BD}_{3}$ in the presence of commodities with equal maximum comparative advantage and demonstrated different results 
each time a different commodity had been chosen. AAA nevertheless has successfully rectified the flaws of Tian's apr-CA model. Cells containing bolded numbers show cases where the AAA has obtained superior optimal value (the sum price of the selected commodities) and/ or price difference (the spread between the maximum and minimum prices of the selected commodities). Detailed processes of commodity distribution of the experimental data and computational results are shown in $<$ Table $2>$ through $<$ Table $12>$. Bolded values in these tables stand for values selected.

Table 1. Comparison of apr-CA model and AAA

\begin{tabular}{|c|c|c|c|c|c|c|c|c|}
\hline \multirow[b]{2}{*}{ Problem } & \multirow[b]{2}{*}{$m * n$} & \multirow{2}{*}{$\begin{array}{c}\text { No. of } \\
\text { Specialized } \\
\text { Commodity }\end{array}$} & \multicolumn{3}{|c|}{ Tian's apr-CA model } & \multicolumn{3}{|c|}{ AAA } \\
\hline & & & $\begin{array}{c}\text { Optimal } \\
\text { Value }\end{array}$ & $\begin{array}{l}\text { Difference of } \\
\text { Prices }\end{array}$ & $\begin{array}{l}\text { Case of } \\
\text { Result }\end{array}$ & $\begin{array}{c}\text { Optimal } \\
\text { Value }\end{array}$ & $\begin{array}{c}\text { Difference } \\
\text { of Prices }\end{array}$ & $\begin{array}{l}\text { Case of } \\
\text { Result }\end{array}$ \\
\hline $\mathrm{BD}_{1}$ & $3 * 3$ & 3 & 14 & 4 & Accept & 14 & 4 & Accept \\
\hline $\mathrm{BD}_{2}$ & $4 * 4$ & 4 & 30 & 15 & Reject & 20 & 2 & Accept \\
\hline $\mathrm{BD}_{3}$ & $4 * 4$ & 4 & 4 & 0 & Accept & 4 & 0 & Accept \\
\hline $\mathrm{BD}_{4}$ & $4 * 4$ & 4 & 19 & 7 & Reject & 16 & 4 & Accept \\
\hline $\mathrm{BD}_{5}$ & $4 * 4$ & 4 & 16 & 6 & Nominal & 16 & 0 & Accept \\
\hline $\mathrm{BD}_{6}$ & $6 * 6$ & 6 & 49 & 7 & Nominal & 49 & 6 & Accept \\
\hline $\mathrm{BD}_{7}$ & $12 * 12$ & 12 & 275 & 61 & Reject & 181 & 35 & Accept \\
\hline \multirow[t]{2}{*}{$\mathrm{UBD}_{1}$} & $2 * 3$ & 2 & 7 & 1 & Accept & 7 & 1 & Accept \\
\hline & & 3 & 11 & 1 & Accept & 11 & 1 & Accept \\
\hline \multirow[t]{2}{*}{$\mathrm{UBD}_{2}$} & $3 * 4$ & 3 & 9 & 4 & Nominal & 9 & 0 & Accept \\
\hline & & 4 & 13 & 4 & Nominal & 13 & 1 & Accept \\
\hline \multirow[t]{2}{*}{$\mathrm{UBD}_{3}$} & $4 * 5$ & 4 & 54 & 12 & Accept & 54 & 12 & Accept \\
\hline & & 5 & 63 & 12 & Accept & 63 & 12 & Accept \\
\hline \multirow[t]{2}{*}{$\mathrm{UBD}_{4}$} & $10 * 13$ & 10 & 182 & 10 & Reject & 178 & 8 & Accept \\
\hline & & 13 & 239 & 10 & Reject & 235 & 7 & Accept \\
\hline
\end{tabular}

Table 2. Optimal commodity distribution for $\mathrm{BD}_{1}$

\begin{tabular}{|c|c|c|c|c|c|}
\hline \multicolumn{5}{|c|}{ Tian's apr-CA model } & \\
\hline \multirow{2}{*}{\multicolumn{2}{|c|}{ Price }} & \multicolumn{3}{|c|}{ Commodity } & \\
\hline & & $a$ & $b$ & $c$ & \\
\hline \multirow{3}{*}{ Country } & $A$ & 4 & 7 & 9 & \\
\hline & $B$ & 2 & 3 & 4 & \\
\hline & $C$ & 3 & 7 & 10 & \\
\hline \multirow[t]{4}{*}{$3 * 3$} & Rate & $a$ & $b$ & $c$ & \multirow{3}{*}{ Minimun } \\
\hline & $A$ & 0.4000 & 0.3500 & 0.3214 & \\
\hline & $B$ & 0.1429 & 0.1071 & 0.1053 & \\
\hline & $C$ & 0.2500 & 0.3500 & 0.3846 & 0.1053 \\
\hline
\end{tabular}

\begin{tabular}{|c|c|c|c|c|c|}
\hline \multicolumn{6}{|l|}{$\overline{\mathrm{AAA}}$} \\
\hline \multirow{2}{*}{\multicolumn{2}{|c|}{ Price }} & \multicolumn{3}{|c|}{ Commodity } & \\
\hline & & $a$ & $b$ & $c$ & \\
\hline \multirow{3}{*}{ Country } & $A$ & 4 & 7 & 9 & $\mathrm{Au}$ \\
\hline & $B$ & 2 & 3 & 4 & Ao \\
\hline & $C$ & 3 & 7 & 10 & An \\
\hline & & 3:- & 01. & $>9:+5$ & \\
\hline
\end{tabular}




\begin{tabular}{|c|c|c|c|c|}
\hline \multirow{2}{*}{\multicolumn{2}{|c|}{ Price }} & \multicolumn{2}{|c|}{ Commodity } & \\
\hline & & $a$ & $b$ & \\
\hline \multirow{2}{*}{ Country } & $A$ & 4 & 7 & \\
\hline & $C$ & 3 & 7 & \\
\hline \multirow[t]{3}{*}{$2 * 2$} & Rate & $a$ & $b$ & \\
\hline & $A$ & 1.3333 & 1.0000 & \\
\hline & $C$ & 0.7500 & 1.0000 & \\
\hline \multirow{2}{*}{\multicolumn{2}{|c|}{ Price }} & $\overline{\text { Commodity }}$ & & \\
\hline & & $b$ & & \\
\hline Country & $A$ & 7 & & \\
\hline \multirow[t]{2}{*}{$1 * 1$} & Price & $b$ & & \\
\hline & $A$ & 7.0000 & & \\
\hline \multicolumn{5}{|c|}{ Optimal Distribution } \\
\hline \multirow{2}{*}{\multicolumn{2}{|c|}{ Price }} & & ommod & \\
\hline & & $a$ & $b$ & $c$ \\
\hline \multirow{3}{*}{ Country } & $A$ & 4 & 7 & 9 \\
\hline & $B$ & 2 & 3 & 4 \\
\hline & $C$ & 3 & 7 & 10 \\
\hline
\end{tabular}

\begin{tabular}{ccccc}
\hline \multirow{2}{*}{ Price } & \multicolumn{3}{c}{ Commodity } \\
\cline { 2 - 5 } Country & $A$ & $a$ & $b$ & $c$ \\
\hline \multirow{3}{*}{ C } & $B$ & 2 & 3 & 9 \\
& $C$ & 3 & 7 & 4 \\
& $C$ &
\end{tabular}

Minimum

0.7500

Minimum

7.0000

Table 3. Optimal commodity distribution for $\mathrm{BD}_{2}$

\begin{tabular}{|c|c|c|c|c|c|}
\hline \multicolumn{6}{|c|}{ Tian's apr-CA model } \\
\hline \multirow{2}{*}{\multicolumn{2}{|c|}{ Price }} & \multicolumn{4}{|c|}{ Commodity } \\
\hline & & $a$ & $b$ & $c$ & $d$ \\
\hline \multirow{4}{*}{ Country } & $A$ & 1 & 2 & 3 & 4 \\
\hline & $B$ & 2 & 4 & 6 & 8 \\
\hline & $C$ & 3 & 6 & 9 & 12 \\
\hline & $D$ & 4 & 8 & 12 & 16 \\
\hline
\end{tabular}

Optimal value, $\mathrm{z}=1+4+9+16=30$

$$
\operatorname{Max}-\operatorname{Min}=16-1=15
$$

\begin{tabular}{|c|c|c|c|c|c|}
\hline$\overline{\mathrm{AAA}}$ & & & & & \\
\hline Dri & & & $\mathrm{Co}$ & odi & \\
\hline Pric & & $a$ & $b$ & $c$ & $d$ \\
\hline & $A$ & 1 & 2 & 3 & 4 \\
\hline Соuntry & $B$ & 2 & 4 & 6 & 8 \\
\hline coumtry & $C$ & 3 & 6 & 9 & 12 \\
\hline & $D$ & 4 & 8 & 12 & 16 \\
\hline
\end{tabular}

Table 4. Optimal commodity distribution for $\mathrm{BD}_{3}$

\begin{tabular}{llllll}
\hline \multicolumn{2}{l}{ Tian's apr-CA model } \\
\multicolumn{1}{c}{ Price } & \multicolumn{4}{c}{ Commodity } \\
\cline { 2 - 6 } & & $a$ & $b$ & $c$ & $d$ \\
\hline \multirow{4}{*}{ Country } & $A$ & 1 & 4 & 3 & 2 \\
& $B$ & 2 & 1 & 4 & 3 \\
& $C$ & 3 & 2 & 1 & 4 \\
& $D$ & 4 & 3 & 2 & 1 \\
\hline
\end{tabular}

Optimal value, $\mathrm{z}=1+1+1+1=4$

$\operatorname{Max}-\operatorname{Min}=1-1=0$

\begin{tabular}{llcccc}
\hline AAA & & \multicolumn{4}{c}{ Commodity } \\
\hline \multirow{2}{*}{ Price } & & \multicolumn{4}{c}{$c$} \\
\cline { 3 - 6 } Country & $B$ & 1 & 4 & 3 & 2 \\
& $A$ & 2 & 1 & 4 & 3 \\
& $C$ & 3 & 2 & 1 & 4 \\
& $D$ & 4 & 3 & 2 & 1 \\
\hline \multirow{4}{*}{ C }
\end{tabular}

Optimal value, $\mathrm{z}=1+1+1+1=4$

$\operatorname{Max}-\operatorname{Min}=1-1=0$ 
Table 5. Optimal commodity distribution for $\mathrm{BD}_{4}$

\begin{tabular}{|c|c|c|c|c|c|}
\hline \multicolumn{6}{|c|}{ Tian's apr-CA model } \\
\hline \multirow{2}{*}{ Price } & & \multicolumn{4}{|c|}{ Commodity } \\
\hline & & $a$ & $b$ & $c$ & $d$ \\
\hline \multirow{4}{*}{ Country } & $\bar{A}$ & 1 & 2 & 3 & 4 \\
\hline & $B$ & 2 & 6 & 12 & 8 \\
\hline & $C$ & 3 & 4 & 9 & 16 \\
\hline & $D$ & 4 & 8 & 6 & 12 \\
\hline
\end{tabular}

Optimal value, $\mathrm{z}=1+8+4+6=19$

$$
\text { Max-Min=8-1=7 }
$$

Table 6. Optimal commodity distribution for $\mathrm{BD}_{5}$

\begin{tabular}{cccccc}
\hline \multirow{2}{*}{ Tian's apr-CA model } & \multicolumn{4}{c}{ Commodity } \\
\hline \multirow{4}{*}{ Price } & \multicolumn{5}{c}{$c$} \\
\cline { 2 - 6 } Country & $A$ & 1 & 2 & 3 & 4 \\
& $B$ & 2 & 3 & 4 & 5 \\
& $C$ & 3 & 4 & 5 & 6 \\
& $D$ & 4 & 5 & 6 & 7 \\
\hline
\end{tabular}

Optimal value, $\mathrm{z}=1+3+5+7=16$

$$
\operatorname{Max}-\mathrm{Min}=7-1=6
$$

\begin{tabular}{|c|c|c|c|c|c|c|c|}
\hline \multicolumn{8}{|c|}{ Tian's apr-CA model } \\
\hline \multirow{2}{*}{\multicolumn{2}{|c|}{ Price }} & \multicolumn{6}{|c|}{ Commodity } \\
\hline & & $a$ & $b$ & $c$ & $d$ & $e$ & $f$ \\
\hline \multirow{6}{*}{ Country } & $A$ & 10 & 9 & 8 & 12 & 9 & 7 \\
\hline & $B$ & 12 & 10 & 7 & 13 & 9 & 8 \\
\hline & $C$ & 8 & 8 & 8 & 14 & 9 & 9 \\
\hline & $D$ & 10 & 7 & 8 & 14 & 8 & 9 \\
\hline & $E$ & 8 & 8 & 8 & 15 & 8 & 9 \\
\hline & $F$ & 12 & 9 & 6 & 14 & 10 & 8 \\
\hline
\end{tabular}

Table 7. Optimal commodity distribution for $\mathrm{BD}_{6}$

Optimal value, $\mathrm{z}=7+13+8+7+8+6=49$

\begin{tabular}{|c|c|c|c|c|c|}
\hline \multicolumn{6}{|l|}{ AAA } \\
\hline \multirow{2}{*}{\multicolumn{2}{|c|}{ Price }} & \multicolumn{4}{|c|}{ Commodity } \\
\hline & & $a$ & $b$ & $c$ & $d$ \\
\hline \multirow{4}{*}{ Country } & $A$ & 1 & 2 & 3 & 4 \\
\hline & $B$ & 2 & 6 & 12 & 8 \\
\hline & $C$ & 3 & 4 & 9 & 16 \\
\hline & $D$ & 4 & 8 & 6 & 12 \\
\hline
\end{tabular}

$\operatorname{Max}-\operatorname{Min}=13-6=7$

\begin{tabular}{|c|c|c|c|c|c|}
\hline \multicolumn{6}{|l|}{$\overline{\mathrm{AAA}}$} \\
\hline \multirow{2}{*}{\multicolumn{2}{|c|}{ Price }} & \multicolumn{4}{|c|}{ Commodity } \\
\hline & & $a$ & $b$ & $c$ & $\bar{d}$ \\
\hline \multirow{4}{*}{ Country } & $A$ & 1 & 2 & 3 & 4 \\
\hline & $B$ & 2 & 3 & 4 & 5 \\
\hline & $C$ & 3 & 4 & 5 & 6 \\
\hline & $D$ & 4 & 5 & 6 & 7 \\
\hline
\end{tabular}

$$
\operatorname{Max}-\operatorname{Min}=6-2=4
$$

Optimal value, $\mathrm{z}=4+4+4+4=16$

$\operatorname{Max}-\mathrm{Min}=4-4=0$

\begin{tabular}{lccccccc}
\hline AAA & \multicolumn{7}{c}{ Commodity } \\
\hline \multirow{2}{*}{ Price } & \multicolumn{7}{c}{$c$} \\
\cline { 2 - 7 } & & $a$ & $b$ & $c$ & $d$ & $e$ & $f$ \\
\hline \multirow{5}{*}{ Country } & $A$ & 10 & 9 & 8 & 12 & 9 & 7 \\
& $B$ & 12 & 10 & 7 & 13 & 9 & 8 \\
& $C$ & 8 & 8 & 8 & 14 & 9 & 9 \\
& $D$ & 10 & 7 & 8 & 14 & 8 & 9 \\
& $E$ & 8 & 8 & 8 & 15 & 8 & 9 \\
& $F$ & 12 & 9 & 6 & 14 & 10 & 8
\end{tabular}

Optimal value, $z=12+8+8+7+8+6=49$

$\operatorname{Max}-\operatorname{Min}=12-6=6$ 
Table 8. Optimal commodity distribution for $\mathrm{BD}_{7}$

\begin{tabular}{|c|c|c|c|c|c|c|c|c|c|c|c|c|c|c|c|c|c|c|c|c|c|c|c|c|c|c|c|}
\hline \multicolumn{14}{|c|}{ Tian's apr-CA model } & \multicolumn{14}{|c|}{ AAA } \\
\hline \multirow{2}{*}{ Price } & \multicolumn{13}{|c|}{ Commodity } & \multirow{2}{*}{ Price } & \multicolumn{13}{|c|}{ Commodity } \\
\hline & & $a$ & $b$ & $c$ & $d$ & $e$ & $f$ & $g$ & $h$ & $i$ & $j$ & $k$ & $l$ & & & $a$ & $b$ & $c$ & $d$ & $e$ & $f$ & $g$ & $h$ & $i$ & $j$ & $k$ & $l$ \\
\hline \multirow{12}{*}{ Country } & $A$ & 79 & 43 & 29 & 88 & 65 & 44 & 35 & 50 & 25 & 68 & 93 & 19 & \multirow{12}{*}{ Country } & $A$ & 79 & 43 & 29 & 88 & 65 & 44 & 35 & 50 & 25 & 68 & 93 & 19 \\
\hline & $B$ & 24 & 59 & 52 & 83 & 90 & 79 & 51 & 12 & 17 & 45 & 36 & 36 & & $B$ & 24 & 59 & 52 & 83 & 90 & 79 & 51 & 12 & 17 & 45 & 36 & 36 \\
\hline & $C$ & 13 & 33 & 26 & 64 & 56 & 86 & 9 & 59 & 39 & 99 & 91 & 5 & & $C$ & 13 & 33 & 26 & 64 & 56 & 86 & 9 & 59 & 39 & 99 & 91 & 5 \\
\hline & $D$ & 53 & 95 & 27 & 72 & 62 & 93 & 91 & 23 & 1 & 1 & 30 & 50 & & $D$ & 53 & 95 & 27 & 72 & 62 & 93 & 91 & 23 & 1 & 1 & 30 & 50 \\
\hline & $E$ & 47 & 55 & 13 & 90 & 53 & 71 & 39 & 23 & 38 & 94 & 44 & 49 & & $E$ & 47 & 55 & 13 & 90 & 53 & 71 & 39 & 23 & 38 & 94 & 44 & 49 \\
\hline & $F$ & 66 & 97 & 33 & 67 & 91 & 7 & 32 & 64 & 63 & 44 & 69 & 94 & & $F$ & 66 & 97 & 33 & 67 & 91 & 7 & 32 & 64 & 63 & 44 & 69 & 94 \\
\hline & $G$ & 85 & 34 & 70 & 27 & 48 & 86 & 3 & 20 & 87 & 99 & 68 & 95 & & $G$ & 85 & 34 & 70 & 27 & 48 & 86 & 3 & 20 & 87 & 99 & 68 & 95 \\
\hline & $H$ & 17 & 55 & 11 & 47 & 23 & 59 & 12 & 94 & 14 & 59 & 67 & 17 & & $H$ & 17 & 55 & 11 & 47 & 23 & 59 & 12 & 94 & 14 & 59 & 67 & 17 \\
\hline & $I$ & 92 & 84 & 71 & 83 & 6 & 17 & 79 & 97 & 4 & 37 & 81 & 63 & & $I$ & 92 & 84 & 71 & 83 & 6 & 17 & 79 & 97 & 4 & 37 & 81 & 63 \\
\hline & $J$ & 47 & 94 & 86 & 62 & 89 & 56 & 25 & 14 & 18 & 18 & 62 & 41 & & $J$ & 47 & 94 & 86 & 62 & 89 & 56 & 25 & 14 & 18 & 18 & 62 & 41 \\
\hline & $K$ & 46 & 26 & 6 & 35 & 49 & 45 & 79 & 11 & 11 & 38 & 66 & 81 & & $K$ & 46 & 26 & 6 & 35 & 49 & 45 & 79 & 11 & 11 & 38 & 66 & 81 \\
\hline & $L$ & 13 & 56 & 76 & 38 & 33 & 59 & 81 & 97 & 45 & 74 & 37 & 1 & & $L$ & 13 & 56 & 76 & 38 & 33 & 59 & 81 & 97 & 45 & 74 & 37 & 1 \\
\hline
\end{tabular}

Optimal value, $\mathrm{z}=43+12+13+1+44+7+3+14+6+62+6+1=275$

Max-Min=62-1=61

Optimal value, $z=25+36+13+1+13+7+27+12+6+14+26+1=181$

Max-Min=36-1=35

Table 9. Optimal commodity distribution for $\mathrm{UBD}_{1}$

\begin{tabular}{ccccc}
\hline Tian's apr-CA model \\
\hline \multirow{2}{*}{ Price } & \multicolumn{4}{c}{ Commodity } \\
\cline { 2 - 5 } & & $a$ & $b$ & $c$ \\
\hline \multirow{2}{*}{ Country } & $A$ & 4 & 7 & 9 \\
& $B$ & 2 & 3 & 4 \\
\hline
\end{tabular}

Optimal value, $\mathrm{z}=4+3+4=11$

$\operatorname{Max}-\mathrm{Min}=4-3=1$

Table 10. Optimal commodity distribution for $\mathrm{UBD}_{2}$

\begin{tabular}{|c|c|c|c|c|c|}
\hline \multicolumn{6}{|c|}{ Tian's apr-CA model } \\
\hline \multirow{2}{*}{\multicolumn{2}{|c|}{ Price }} & \multicolumn{4}{|c|}{ Commodity } \\
\hline & & $a$ & $b$ & $c$ & $d$ \\
\hline \multirow{3}{*}{ Country } & $\bar{A}$ & 1 & 2 & 3 & 4 \\
\hline & $B$ & 2 & 3 & 4 & 5 \\
\hline & $C$ & 3 & 4 & 5 & 6 \\
\hline
\end{tabular}

Optimal value, $\mathrm{z}=1+4+3+5=13$

$\operatorname{Max}-\operatorname{Min}=5-1=4$

\begin{tabular}{llccc}
\hline AAA & \multicolumn{4}{c}{ Commodity } \\
\hline \multirow{2}{*}{ Price } & & \multicolumn{3}{c}{$c$} \\
\cline { 2 - 5 } Country & $A$ & 4 & 7 & 9 \\
& $B$ & 2 & 3 & 4 \\
\hline
\end{tabular}

Optimal value, $\mathrm{z}=4+3=7$

Max-Min=4-3=1

\begin{tabular}{lccccc}
\hline Proposed AAA & \multicolumn{4}{c}{ Commodity } \\
\hline \multirow{2}{*}{ Price } & & $a$ & $b$ & $c$ & $d$ \\
\cline { 2 - 6 } Country & $A$ & 1 & 2 & 3 & 4 \\
& $B$ & 2 & 3 & 4 & 5 \\
& $C$ & 3 & 4 & 5 & 6 \\
\hline \multicolumn{4}{c}{ Optimal value, $\mathrm{z}=3+3+3=9$}
\end{tabular}

$\operatorname{Max}-\operatorname{Min}=3-3=0$

Table 11.Optimal commodity distribution for $\mathrm{UBD}_{3}$

\begin{tabular}{ccccccc}
\hline Tian's apr-CA model \\
\hline \multirow{2}{*}{ Price } & \multicolumn{6}{c}{ Commodity } \\
\cline { 2 - 6 } & & $a$ & $b$ & $c$ & $d$ & $e$ \\
\hline \multirow{4}{*}{ Country } & $A$ & 7 & 9 & 10 & 10 \\
& $B$ & 14 & 17 & 18 & 12 & 15 \\
& $C$ & 19 & 20 & 21 & 18 & 21 \\
& $D$ & 15 & 19 & 18 & 19 & 16 \\
\hline
\end{tabular}

Optimal value, $\mathrm{z}=7+9+12+19+16=63$

Max-Min=19-7=12

\begin{tabular}{llccccc}
\hline AAA & \multicolumn{6}{c}{ Commodity } \\
\hline \multirow{2}{*}{ Price } & \multicolumn{5}{c}{$c$} \\
\cline { 2 - 7 } Country & $A$ & 9 & 7 & 9 & 10 & 10 \\
& $B$ & 14 & 17 & 18 & 12 & 15 \\
& $C$ & 19 & 20 & 21 & 18 & 21 \\
& $D$ & 15 & 19 & 18 & 19 & 16 \\
\hline
\end{tabular}

Optimal value, $\mathrm{z}=7+12+19+16=54$

Max-Min=19-7=12 
Table 12. Optimal commodity distribution for $\mathrm{UBD}_{4}$

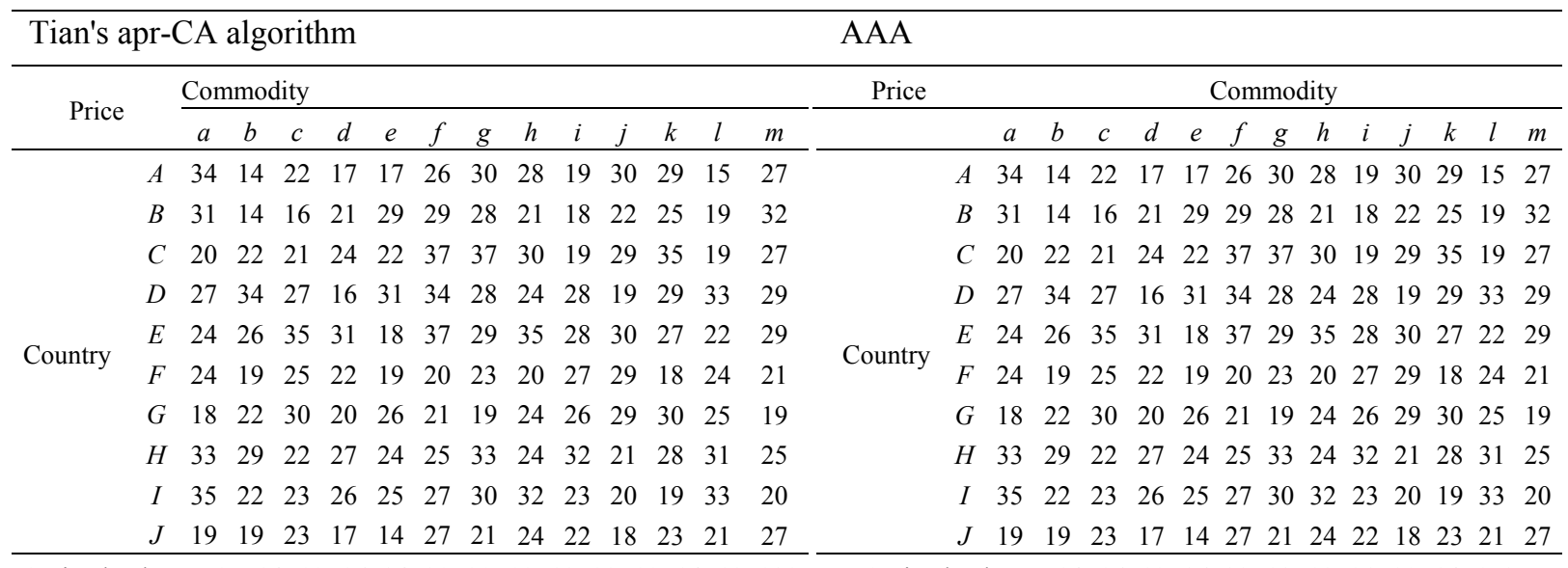

Optimal value, $\mathrm{z}=15+14+19+16+24+20+20+19+19+22+19+14+18=239$

\section{Concluding Remarks}

In international trade, all participant countries can gain from specializing in the production of a good and trading it for another. Ricardo's comparative advantage model has provided a commodity distribution method for 2-country, 2-commodity case. Tian (2008) has generalized this model into a multi-country, multi-commodity model by taking average price rates in lieu of sum price rates. This algorithm nevertheless occasionally fails to achieve optimal commodity distribution or to facilitate international trade even if it succeeds in optimal distribution for the price difference - difference between the maximum price and the minimum price of the selected commodities - is large. Moreover, in the existence of multiple commodities with equal maximum comparative advantage, it yields different results depending on the commodity it selects. To remedy these shortcomings of Tian's model (Tian, 2008), this paper proposes a simple algorithm that selects a commodity for each country based on their absolute advantage (prices) instead of comparative advantage (price rates). Whereas Tian's model (Tian, 2008) repeatedly computes average price rates (apr) and selects the maximum comparative advantage (the smallest average price rate), the proposed algorithm simply selects a commodity with the maximum absolute advantage (minimum price) for each country and reassigns commodities of over-assigned countries to under-assigned countries. When applied to various balanced-distribution and unbalanced-distribution case data, the proposed AAA is found to yield results superior to those of Tian's apr-CA model through a much simpler process.

\section{References}

Baumol, W. J., \& Binder, A. S. (2009). Economics: Principles and Policy. South-Western Cengage Learning.

Deardorff, A. V. (2005). How Robust is Comparative Advantage. Review of International Economics, 13(5), 1004-1016. http://dx.doi.org/10.1111/j.1467-9396.2005.00552.x

Irving, R. W. (2008). Stable Matching Problems with Exchange Restrictions. Journal of Combinatorial Optimization, 16, 344-360. http://dx.doi.org/10.1007/s10878-008-9153-1

Krugman, P. (2010). A Globalization Puzzle. New York Times. Retrieved from http://krugman.blogs.nytimes.com/2010/02/21/a-globalization-puzzle/

Ntaimo, L. (2005). Transportation and Assignment Problems [PowerPoint slides]. Retrieved from http://www.learningace.com/doc/2243668/64b4e81d672f648fbd579b8e5fe2c2d7/chapter-7

O'Sullivan, A., \& Sheffrin, S. M. (2003). Economics: Models in Action. Prentice Hall: Addison Wesley Longman.

Parchure, R. (2011). On Graham's Theory of Multicountry Multicommodity Trade. Working Paper No. 23, Gokhale Institute of Politics and Economics. Retrieved from http://dspace.gipe.ac.in/jspui/bitstream/1/502/1/wp23.pdf

Ricardo, D. (1817). On the Models of Political Economy and Taxation. J. M. Dent \& Sons, Ltd.

Tian, Y. (2008). A New Idea about Ricardo's Comparative Advantage: Theory on Condition of Multi-Commodity and Multi-Country. International Journal of Business and Management, 3(12), 155-160. Retrieved from 
http://www.ccsenet.org/journal/index.php/ijbm/article/view/771/745

\section{Copyrights}

Copyright for this article is retained by the author(s), with first publication rights granted to the journal.

This is an open-access article distributed under the terms and conditions of the Creative Commons Attribution license (http://creativecommons.org/licenses/by/3.0/). 